\title{
Lipid profile and insulin sensitivity in rats fed with high-fat or high-fructose diets
}

\author{
Muhammad-Quaid Zaman ${ }^{1,2}$, Véronique Leray ${ }^{1,2}$, Jérôme Le Bloc'h ${ }^{1,2,3}$, Chantal Thorin ${ }^{4}$, \\ Khadija Ouguerram ${ }^{2,3}$ and Patrick Nguyen ${ }^{1,2 *}$ \\ ${ }^{1}$ LUNAM Université, Oniris, National College of Veterinary Medicine, Food Science and Engineering, Nutrition and \\ Endocrinology Unit, PO Box 40706, F-44307 Nantes, Cedex 3, France \\ ${ }^{2}$ CRNH, Human Nutrition Research Center of Nantes, CHU, F-44093 Nantes, France \\ ${ }^{3}$ INSERM, UMR 915, Institute of Thorax, Nantes F-44000, France \\ ${ }^{4}$ LUNAM Université, Oniris, National College of Veterinary Medicine, Food Science and Engineering, Statistics Unit, \\ PO Box 40706, F-44307 Nantes, Cedex 3, France
}

(Received 16 November 2010 - Revised 29 June 2011 - Accepted 3 July 2011)

\section{Abstract}

The occurrence and severity of obesity- and insulin resistance-related disorders vary according to the diet. The aim of the present longitudinal study was to examine the effects of a high-fat or a high-fructose diet on body weight (BW), body fat mass, insulin sensitivity (IS) and lipid profiles in a rat model of dietary-induced obesity and low IS. A total of eighteen, 12-week-old male Wistar rats were divided into three groups, and were fed with a control, a high-fat (65\% lipid energy) or a high-fructose diet (65\% fructose energy) for 10 weeks. BW, body fat mass $\left({ }^{2} \mathrm{H}_{2} \mathrm{O}\right.$ dilution method), IS (euglycaemic-hyperinsulinaemic clamp technique), plasma glucose, insulin, NEFA, TAG and total cholesterol were assessed before and at the end of 10-week period. Cholesterol was measured in plasma lipoproteins separated from pooled samples of each group and each time period by using fast-protein liquid chromatography. All rats had similar BW at the end of the 10-week period. Body fat mass was higher in the high-fat group compared to the control group. There was no change in basal glycaemia and insulinaemia. The IS was lower in the high-fat group and was unchanged in the high-fructose group, compared to the control group. Plasma TAG concentration and cholesterol distribution in lipoproteins did not change over time in any group. Plasma NEFA concentration decreased, whereas plasma TAG concentration increased over time, regardless of the diet in both cases. The 10-week high-fat diet led to obesity and low IS, whereas rats fed with the high-fructose diet exhibited no change in IS and lipidaemia. The high-fat diet had more deleterious response than high-fructose diet to induce obesity and low IS in rats.

Key words: insulin resistance: obesity: dyslipidemia: high-fat diet: high-fructose diet: rat

There is a growing prevalence of obesity and low insulin sensitivity (IS), often called insulin resistance, in human subjects. Thus, there is a need for an animal model to study the time course of these metabolic disturbances as well as their unhealthy consequences. Different animal models have been used to study obesity and IS, notably the rat, in which obesity can be caused by genetic mutations or induced by nutritional interventions. As human obesity is mainly due to nutritional habits, animal models of obesity and low IS induced by specific diets may be preferable to genetic models.

Various nutritional interventions have been used to induce obesity, low IS and dyslipidaemia in rats. High-fat diets have been shown to cause these metabolic disorders in previous studies, but there has been a large variability in the intensity of the metabolic changes ${ }^{(1-5)}$. High-fructose diets have also been shown to lower IS and promote mild-to-severe dyslipidaemia $^{(6-9)}$. The differences in nutritional interventions, such as diet composition and interventional duration, have complicated the comparisons of these studies. Therefore, it is difficult to define the best nutritional intervention to induce obesity in an animal model that closely mimics the human disease. Longitudinal studies could be useful in determining the best diet to induce obesity and related disorders. To our knowledge, this type of approach has never been conducted in rats. Here, we aimed to perform a longitudinal study to compare the effects of a high-fat diet and a high-fructose diet on body weight (BW), IS and plasma lipid profiles in rats.

Abbreviations: BW, body weight; IS, insulin sensitivity.

*Corresponding author: Professor P. Nguyen, fax +3324068 77 46, email patrick.nguyen@oniris-nantes.fr 


\section{Materials and methods}

\section{Animal groups and diets}

Male Wistar rats (12 weeks old; Janvier, Le Genest Saint-Isle, France) were randomly separated into three groups (six per group): control, high fat or high fructose. According to their groupings, the rats were fed with a control diet $39.7 \%$ maize starch, 20\% dextrose, 5.8\% sunflower oil and 20.5\% casein by weight), a high-fat diet ( $12.7 \%$ maize starch, $6.5 \%$ dextrose, $3.9 \%$ sunflower oil, $31.3 \%$ lard and $28.6 \%$ casein by weight) or a high-fructose diet (59.7\% fructose, $5 \cdot 8 \%$ sunflower oil and $20.5 \%$ casein by weight) for 10 weeks. The rats were housed in individual cages, with free access to the feed and water. The rats were maintained under a 12-h light-12-h dark cycle and a temperature of $22 \pm 2^{\circ} \mathrm{C}$. The animals were housed at Oniris (National College of Veterinary Medicine, Food Science and Engineering, Nantes, France), according to the regulations for animal welfare of the French Ministry of Agriculture. The experimental protocol adhered to the European Union guidelines and was approved by the local animal use and care advisory committee.

\section{Body weight and body fat mass}

$\mathrm{BW}$ was recorded weekly. The body fat mass was determined by isotope dilution $\left({ }^{2} \mathrm{H}_{2} \mathrm{O}\right.$; Eurisotop, Gif-sur-Yvette, France) on week 1 (before the dietary intervention) and at the end of the 10-week diet period (week 11). Blood samples (1 ml) were collected before and $2 \mathrm{~h}$ after a ${ }^{2} \mathrm{H}_{2} \mathrm{O}$ injection ( $500 \mathrm{mg} / \mathrm{kg} \mathrm{BW}$ ). Plasma ${ }^{2} \mathrm{H}_{2} \mathrm{O}$ concentrations were measured using Fourier-transformed IR spectroscopy (Brüker SA, Wissembourg, France).

\section{Euglycaemic-hyperinsulinaemic clamp technique}

The euglycaemic-hyperinsulinaemic clamp technique was performed before and at the end of the 10-week diet period. The catheter was inserted under anaesthesia into the jugular vein of an animal that was not fed overnight. Insulin (Actrapid; Novo Nordisk, Bagsvaerd, Denmark) was perfused $[72 \mathrm{mU} / \mathrm{kg}$ ( $500 \mathrm{pmol} / \mathrm{kg}$ ) for $1 \mathrm{~min}$, then $18 \mathrm{mU} / \mathrm{kg}$ per min $(125 \mathrm{pmol} / \mathrm{kg}$ per $\min$ ) for $3 \mathrm{~h}$ ] and glycaemia was measured at every $5 \mathrm{~min}$. Glucose (20\%; Braun Medical SAS, Boulogne Cedex, France) was perfused at a variable rate. The glucose infusion rate (mg/kg per min) was adjusted to attain and maintain the basal glycaemia. In hyperinsulinaemic conditions, glucose infusion rate measures the insulin-mediated glucose uptake and is considered as a good reflection of IS. Glycaemia was measured using the glucose oxidase method (Accu-Chek Active; Roche Diagnostics, Mannheim, Germany). Insulin was measured using ELISA (Rat Insulin; Shibayagi Company Limited, Gunma, Japan).

\section{Plasma lipid profiles}

The basal plasma concentrations of total cholesterol, NEFA and TAG were assayed before and at the end of the 10-week diet period using enzymatic methods (Cholestérol 
RTU, BioMérieux, Marcy-l'Etoile, France; Triglycérides enzymatique TG PAP150, BioMérieux; and NEFA C, Wako, Oxoid, Dardilly, France).

The plasma samples were pooled for each group and each time period (weeks 1 and 11), and the plasma lipoproteins were separated using a fast-protein liquid chromatography system (UNICORN 520; GE Healthcare, Pittsburgh, PA, USA). The cholesterol concentration was measured in each fraction.

\section{Statistical analysis}

Data analysis was performed using Statview software (version 5.0; SAS Institute Inc., Cary, NC, USA) and R software (version 2.10, lme4 package; R Foundation for Statistical Computing, Vienna, Austria). Data were expressed as mean values with the standard error of the mean. A linear mixed-effects model has been performed in order to study the effect of time, type of diet and the interaction between them for each variable. The mixed-effects models are the most efficient way to analyse repeated measurements data ${ }^{(10)}$. A multiple comparison of means procedure with Tukey contrasts, adapted to the mixed-effects models, has been used when the interactions between time and the type of diet were significant. A significant difference has been considered for $P$ value $<0 \cdot 05$.

\section{Results}

\section{Body weight and body fat mass}

Table 1 gives the values of BW and body fat mass of the control, the high-fat and the high-fructose groups before and at the end of the 10 -week period. The BW gain was similar for all groups. At week 11, the high-fat group had significantly higher $(P<0.05)$ body fat mass compared to the control group. At week 11, compared to initial values from week 1, the high-fat diet caused a 393 (sEm 170) \% increase in body fat mass and high-fructose diet caused a 139 (SEM 23)\% increase.

\section{Insulin sensitivity}

No differences were observed in basal glycaemia and insulinaemia among groups. The high-fat group had lower IS compared to the control group at week 11, assessed by significantly lower $(P<0.05)$ glucose infusion rate value. The high-fructose group had no difference in IS compared to the control group (Table 1).

\section{Plasma lipid profiles}

Plasma TAG concentration was not different among groups at any time. Plasma NEFA concentration decreased over time in all groups, regardless of the diet.

At week 1, the plasma total cholesterol concentration was different among groups. Indeed, it was higher in the control group than in others. There was a 38 (SEM 12)\% increase in total cholesterol concentration in rats fed with high-fat diet and a 15 (SEM 8)\% increase in rats fed with high-fructose diet, but the difference did not reach level of significance (Table 1). Nevertheless, plasma total cholesterol concentration increased over time, regardless of the diet.

On the basis of a pooled sample from each of the groups, there is a preliminary indication that there was no difference due to diet (data not shown) in the plasma lipoprotein fractions, but these results must be considered to be very preliminary.

\section{Discussion}

The aim of the present study was to compare the effects of a high-fat and a high-fructose diet on obesity-related disturbances in rats by using a longitudinal approach. In the present study, all the basal values were in the range of normal values of rats. In the literature, various concentrations of plasma lipids have been measured in control rats. However, we did not find any study of obesity and IS in rats with the baseline values of all variables. Therefore, we used a longitudinal approach and composed the groups randomly. The plasma total cholesterol concentration was significantly different among groups at baseline level (week 1). The randomisation process used to compose the groups had been expected to abolish all the differences among groups, and all cholesterol concentrations have been measured at the same time, i.e. at the end of the study, to guarantee same analytical conditions. Unfortunately, the randomisation process failed to reach its objectives, at least in this regard (there was no other difference among the groups at week 1). The baseline values were then taken into account in the statistical analysis in order to nullify the pre-existing differences. Another factor is the age at which exposure to a high-fat or high-fructose diet started. It has been shown that adult rats fed with high-fructose diet produce signs of metabolic syndrome, but young rats do not ${ }^{(11)}$. We therefore chose adult rats to conduct the present experiment.

We observed similar BW at the end of the 10-week period in all groups. However, rats fed with high-fat diet had higher body fat mass compared to the control rats. Increase in adiposity has previously been described in rats fed with a highfat diet ${ }^{(2-5,12)}$, and it was associated with increase in weight gain. An increase in BW alone does not necessarily represent obesity, but other factors have to be taken into account, such as changes in body composition. In the present study, the increase in body fat mass reflected the obesogenic property of the high-fat diet, which was due to lard being used as the source of dietary fat. This excess fat was stored in the form of adipose tissue in the body. In rats fed with high-fructose diet, we observed no change in BW and body fat mass compared to the control rats. Previous studies showed that administering high-fructose ( $60 \%$ by weight) diet for about 10 weeks increased $\mathrm{BW}^{(6,8,13)}$ and epididymal and retroperitoneal fat depots $^{(13)}$. However, de Moura et al. ${ }^{(11)}$ observed no difference in BW in adult rats administered fructose, despite higher retroperitoneal, mesenteric and subcutaneous fat depots weights. This suggests that high-fructose diet does not necessarily lead to obesity.

The rats fed with high-fat diet had lower IS (assessed by glucose infusion rate, which is considered as a good reflection of 
IS, as it measures the insulin-mediated glucose uptake under hyperinsulinaemic conditions) compared to the control group, as previously shown in several studies ${ }^{(14,15)}$. Moreover, low IS has been shown to be associated with an increase in epididymal $\mathrm{fat}^{(3,4)}$, and the present results of higher body fat mass in rats fed with high-fat diet are consistent with the previous results. However, basal glycaemia and insulinaemia were unchanged. Some studies using high-fat diet (lard or safflower oil) reported low IS, associated with hyperinsulinaemia $^{(3-5,12,16)}$ and hyperglycaemia ${ }^{(16)}$. We think that we could have assessed low IS by euglycaemic-hyperinsulinaemic clamp technique before the appearance of hyperglycaemic and hyperinsulinaemic states, and that the present experiment could have been long enough to develop insulin resistance but not diabetes.

On the other hand, we found no change in IS, basal glycaemia and insulinaemia in rats fed with high-fructose diet. Many previous studies have reported hyperglycaemia ${ }^{(7-9,13)}$, but Nakagawa et al. ${ }^{(6)}$ did not show any modification in glycaemia after administering high-fructose diet. The higher hepatic glycogen content that has been described in rats fed with high-fructose diet ${ }^{(17)}$ could prevent hyperglycaemia. Previous studies have also shown that the high-fructose diet developed low IS and was associated with increased plasma TAG and NEFA concentrations ${ }^{(6-8)}$. These increased concentrations in response to high-fructose diet could have an important role in the development of low IS by reducing insulin signalling pathway (reviewed in Tappy \& Le ${ }^{(18)}$ ). However, we did not find dyslipidaemia, which is in accordance with the unchanged IS. Moreover, in a study in rats, high-sucrose diet has been shown to induce low IS in the liver before muscle $^{(19)}$. We assessed the IS by using the euglycaemichyperinsulinaemic clamp technique, which is the gold standard method for direct assessment of the whole-body $\mathrm{IS}^{(20)}$. We suggest that high-fructose diet could have caused minor impairment of insulin action in the liver, but could not have caused whole-body low IS.

We observed no difference in plasma total cholesterol concentration between the groups, but there was a significant increase over time, regardless of the diet. This increase could have reflected an effect of age, whereas the absence of dietary cholesterol could be a possible explanation for the absence of any specific effect of the diet. Indeed, additional dietary fat, whatever its nature, had no effect on plasma cholesterol concentration in the absence of dietary cholesterol in a study conducted in hamsters ${ }^{(21)}$. In the present study, the non-significant increase in plasma total cholesterol in rats fed with high-fat diet could be due to small amount of dietary cholesterol in lard. On the other hand, previous studies reported variable responses of the high-fructose diets on plasma total cholesterol concentration ${ }^{(7,8,13,22)}$, and even the same high-fructose diets behaved differently ${ }^{(7,13)}$. Thus, it is difficult to conclude about the effects of fructose diet on cholesterol concentration. Moreover, the dietary cholesterol and amount of fat in a high-fructose diet could affect plasma cholesterol concentration. We also observed no difference in cholesterol concentrations in plasma lipoproteins, as reported by Sinitskaya et $a l{ }^{(2)}$. Conversely, Mohamed Salih et al. ${ }^{(8)}$ reported an increase in VLDL-cholesterol and LDL-cholesterol, and a decrease in HDL-cholesterol at the end of a high-fructose diet. The present results for lipoprotein-cholesterol concentrations are in accordance to unchanged plasma total cholesterol concentration, and absence of dietary cholesterol could possibly be the cause for unchanged cholesterol concentration.

Plasma basal NEFA concentration was not different among groups. Variable changes in plasma NEFA concentration have been described in response to a high-fat diet $^{(1,2,12)}$. The present results in rats fed with high-fructose diet were not consistent with some previous reports ${ }^{(8,13)}$. We hypothesise that the liver could have higher hepatic TAG storage ${ }^{(23)}$, by capturing a bulk amount of plasma NEFA, which would explain that no difference in plasma NEFA concentration was observed.

We found no change in plasma basal TAG concentration in any group. Some studies showed an increase in plasma TAG concentration at the end of a high-fat $\operatorname{diet}^{(3,4)}$, whereas others with nearly the same fat content did not ${ }^{(1,2,5)}$. Variable response on plasma basal TAG concentration has also been reported in rats fed with high-fructose diet ${ }^{(6-8,13,22,24)}$. A few studies have reported higher postprandial TAG concentration in rats fed with high-fructose diet, but no change in the basal TAG concentration for a short period $(2 \text { weeks })^{(25)}$ or for a long period (11 months) ${ }^{(26)}$. In the present study, we measured only the basal TAG concentration. We suggest that the rats in our study could have had higher postprandial TAG concentration that could not have been observed in overnight unfed rats.

On the basis of the present findings, feeding 12-week-old rats a high-fat diet for 10 weeks induces obesity and low IS. In contrast, the high-fructose diet produced no change in obesity-related disorders. In the future, it will be useful to study the specific effects of different fats, oils or fatty acids that account for the discrepancy between these studies.

\section{Acknowledgements}

The financial support in the form of doctoral fellowship to M.-Q. Z. from the Higher Education Commission, Pakistan, is greatly acknowledged. The authors are grateful to Philippe Bleis and Samuel Ninet for their technical assistance and for taking care of the animals. The present study was designed by P. N., V. L., K. O. and M.-Q. Z. and supervised by P. N. and V. L; M.-Q. Z. performed the experiments; C. T. and M.-Q. Z. conducted the statistical analysis; M.-Q. Z. and J. L. B. wrote the manuscript; and P. N., V. L., K. O. and J. L. B. critically reviewed the manuscript. The authors declare no conflicts of interest.

\section{References}

1. Morens C, Sirot V, Scheurink AJ, et al. (2006) Low-carbohydrate diets affect energy balance and fuel homeostasis differentially in lean and obese rats. Am J Physiol Regul Integr Comp Physiol 291, R1622-R1629. 
2. Sinitskaya N, Gourmelen S, Schuster-Klein C, et al. (2007) Increasing the fat-to-carbohydrate ratio in a high-fat diet prevents the development of obesity but not a prediabetic state in rats. Clin Sci (Lond) 113, 417-425.

3. Sridhar MG, Vinayagamoorthi R, Arul Suyambunathan V, et al. (2008) (2008) Bitter gourd (Momordica charantia) improves insulin sensitivity by increasing skeletal muscle insulin-stimulated IRS-1 tyrosine phosphorylation in highfat-fed rats. Br J Nutr 99, 806-812.

4. Vinayagamoorthi R, Bobby Z \& Sridhar MG (2008) Antioxidants preserve redox balance and inhibit c-Jun-N-terminal kinase pathway while improving insulin signaling in fat-fed rats: evidence for the role of oxidative stress on IRS-1 serine phosphorylation and insulin resistance. J Endocrinol 197, 287-296.

5. Matveyenko AV, Gurlo T, Daval M, et al. (2009) Successful versus failed adaptation to high-fat diet-induced insulin resistance: the role of IAPP-induced beta-cell endoplasmic reticulum stress. Diabetes 58, 906-916.

6. Nakagawa T, Hu H, Zharikov S, et al. (2006) A causal role for uric acid in fructose-induced metabolic syndrome. $A m \mathrm{~J}$ Physiol Renal Physiol 290, F625-F631.

7. Liu IM, Tzeng TF \& Liou SS (2011) A Chinese herbal decoction, Dang Gui Bu Xue Tang, prepared from Radix Astragali and Radix Angelicae sinensis, ameliorates insulin resistance induced by a high-fructose diet in rats. Evid Based Complement Alternat Med, vol. 2011, Article ID 248231, 11 pages. doi:10.1093/ecam/nep004.

8. Mohamed Salih S, Nallasamy P, Muniyandi P, et al. (2009) Genistein improves liver function and attenuates non-alcoholic fatty liver disease in a rat model of insulin resistance. J Diabetes 1, 278-287.

9. Kannappan S, Palanisamy N \& Anuradha CV (2010) Suppression of hepatic oxidative events and regulation of eNOS expression in the liver by naringenin in fructose-administered rats. Eur J Pharmacol 645, 177-184.

10. Davidian M \& Giltinan DM (1995) Nonlinear Models for Repeated Measurement Data. New York, NY: Chapman \& Hall/CRC.

11. de Moura RF, Ribeiro C, de Oliveira JA, et al. (2009) Metabolic syndrome signs in Wistar rats submitted to different high-fructose ingestion protocols. Br J Nutr 101, 1178-1184.

12. Posey KA, Clegg DJ, Printz RL, et al. (2009) Hypothalamic proinflammatory lipid accumulation, inflammation, and insulin resistance in rats fed a high-fat diet. Am J Physiol Endocrinol Metab 296, E1003-E1012.
13. Shih CC, Lin CH, Lin WL, et al. (2009) Momordica charantia extract on insulin resistance and the skeletal muscle GLUT4 protein in fructose-fed rats. J Ethnopharmacol 123, 82-90.

14. Kraegen EW, Clark PW, Jenkins AB, et al. (1991) Development of muscle insulin resistance after liver insulin resistance in high-fat-fed rats. Diabetes 40, 1397-1403.

15. Oakes ND, Cooney GJ, Camilleri S, et al. (1997) Mechanisms of liver and muscle insulin resistance induced by chronic high-fat feeding. Diabetes 46, 1768-1774.

16. Vikram A, Jena GB \& Ramarao P (2010) Increased cell proliferation and contractility of prostate in insulin resistant rats: linking hyperinsulinemia with benign prostate hyperplasia. Prostate 70, 79-89.

17. Yadav H, Jain S, Yadav M, et al. (2009) Epigenomic derangement of hepatic glucose metabolism by feeding of high fructose diet and its prevention by Rosiglitazone in rats. Dig Liver Dis 41, 500-508.

18. Tappy L \& Le KA (2010) Metabolic effects of fructose and the worldwide increase in obesity. Physiol Rev 90, 23-46.

19. Pagliassotti MJ, Prach PA, Koppenhafer TA, et al. (1996) Changes in insulin action, triglycerides, and lipid composition during sucrose feeding in rats. Am J Physiol 271, R1319-R1326.

20. DeFronzo RA, Tobin JD \& Andres R (1979) Glucose clamp technique: a method for quantifying insulin secretion and resistance. Am J Physiol 237, E214-E223.

21. Sessions VA \& Salter AM (1994) The effects of different dietary fats and cholesterol on serum lipoprotein concentrations in hamsters. Biochim Biophys Acta 1211, 207-214.

22. Stark AH, Timar B \& Madar Z (2000) Adaptation of SpragueDawley rats to long-term feeding of high fat or high fructose diets. Eur J Nutr 39, 229-234.

23. Benhizia F, Hainault I, Serougne C, et al. (1994) Effects of a fish oil-lard diet on rat plasma lipoproteins, liver FAS, and lipolytic enzymes. Am J Physiol 267, E975-E982.

24. Rizkalla SW, Boillot J, Tricottet V, et al. (1993) Effects of chronic dietary fructose with and without copper supplementation on glycaemic control, adiposity, insulin binding to adipocytes and glomerular basement membrane thickness in normal rats. Br J Nutr 70, 199-209.

25. Koo HY, Wallig MA, Chung BH, et al. (2008) Dietary fructose induces a wide range of genes with distinct shift in carbohydrate and lipid metabolism in fed and fasted rat liver. Biochim Biophys Acta 1782, 341-348.

26. Lee YC, Ko YH, Hsu YP, et al. (2006) Plasma leptin response to oral glucose tolerance and fasting/re-feeding tests in rats with fructose-induced metabolic derangements. Life Sci $\mathbf{7 8}$, 1155-1162. 